\title{
The Diffusion of Self-Avoiding Random Walk in High Dimensions
}

\author{
Gordon Slade* \\ Department of Mathematics, University of Virginia, Charlottesville, VA 22903, USA
}

\begin{abstract}
We use the Brydges-Spencer lace expansion to prove that the mean square displacement of a $T$ step strictly self-avoiding random walk in the $d$ dimensional square lattice is asymptotically of the form $D T$ as $T$ approaches infinity, if $d$ is sufficiently large. The diffusion constant $D$ is greater than one.
\end{abstract}

\section{Introduction}

A $T$ step self-avoiding walk on the $d$ dimensional square lattice $\mathbb{Z}^{d}$ is a set of $T+1$ points $\omega(0)=0, \omega(1), \omega(2), \ldots, \omega(T)$ in $\mathbb{Z}^{d}$ with $|\omega(i+1)-\omega(i)|=1$ and $\omega(i) \neq \omega(j)$ for $i \neq j$. A probability measure is defined on the set of all $T$ step self-avoiding walks by assigning an equal probability to each such walk. Numerical and other evidence suggests that the mean square displacement with respect to this measure, i.e., the expected value $\left\langle\omega(T)^{2}\right\rangle$ of $\omega(T) \cdot \omega(T)$, is asymptotically of the form $D T^{\alpha}$ as $T \rightarrow \infty$, where $\alpha=1.5$ for $d=2, \alpha=1.18$ for $d=3, \alpha=1$ with logarithmic corrections for $d=4$, and $\alpha=1$ for $d \geqq 5$ [4]. For $d=1$ there are only two self-avoiding walks, $\left\langle\omega(T)^{2}\right\rangle=T^{2}$, and $\alpha=2$. Removing the self-avoidance constraint $\omega(i) \neq \omega(j), i \neq j$ gives the simple random walk, for which $\left\langle\omega(T)^{2}\right\rangle=T$ in all dimensions.

In spite of the apparent simplicity of the self-avoiding walk model, apart from the result obtained below there is no rigorous proof that $\alpha$ is as stated above. In this paper we prove that $\alpha=1$ and $D>1$ for $d \geqq d_{0}$, for some $d_{0} \geqq 5$. No effort has been made to obtain the best possible value of $d_{0}$. It is not surprising that $D>1$ here, since it is to be expected that a self-avoiding walk will on the average end up farther away from the origin than a simple walk.

Other results for the critical exponents of self-avoiding random walk can be found in $[7,8]$. In [8] the connection between self-avoiding walk and quantum field theory is also explained. Lawler [6] considered a related model, the looperased self-avoiding random walk, and proved that for $d \geqq 4$ scaled loop-erased

\footnotetext{
* Present address: Department of Mathematics and Statistics, McMaster University, Hamilton, Ontario, Canada L8S 4K1
} 
walk converges in distribution to Brownian motion. In particular, $\alpha=1$ for $d \geqq 4$ (with logarithmic corrections for $d=4$ ) in this model. Brydges and Spencer [3,1] used their lace expansion to show that $\alpha=1$ for $d \geqq 5$ for weakly self-avoiding random walk, for which self-intersections are not forbidden but rather discouraged by a small probability penalty.

The weakly self-avoiding walk was studied in [3] by exploiting the fact that it is a small perturbation of simple random walk. But in high dimensions the strictly self-avoiding random walk is also a small perturbation of simple random walk. A result in this spirit was obtained by Kesten [5], who showed that in high dimensions the main effect of the constraint that a walk be self-avoiding is the exclusion of immediate reversals. In this paper we apply the Brydges-Spencer lace expansion to the strictly self-avoiding walk in high dimensions, obtaining convergence of the expansion by taking $d$ to be large rather than by taking the probability penalty associated with self-intersections to be small as in [3]. We use a simplified proof of convergence of the expansion, avoiding the intricate induction argument used in [3]. To help make this paper self-contained a derivation of the lace expansion is given in the next section.

We now introduce the notation. We begin by considering walks which have no self-intersections on any time interval of length less than a memory $\tau$. That is, we consider $T$ step nearest-neighbour walks $\omega$ whose probability is proportional to

$$
\prod_{s t \in \mathscr{B}_{r}([0, T])}\left(1+U_{s t}(\omega)\right)
$$

where for an interval $I$ of positive integers

$$
\mathscr{B}_{\tau}(I)=\{s t: s<t,|s-t| \leqq \tau, s, t \in I\},
$$

and $U_{s t}(\omega)=-1$ if $\omega(s)=\omega(t)$ and equals zero otherwise. For $\tau=0$ this is simple random walk while for $\tau \geqq T$ it is strictly self-avoiding walk. For $x \in \mathbb{Z}^{d}$, let

$$
N_{\tau}(x, T)=(2 d)^{-T} \sum_{\substack{\omega,|\omega|=T \\ \omega(T)=x}} \prod_{s t \in \mathscr{B}_{\tau}[(0, T])}\left(1+U_{s t}(\omega)\right)
$$

We set $N_{\tau}(x, 0)=\delta_{x, 0}$. The following transforms of $N_{\tau}(x, T)$ are distinguished from one another by their arguments:

$$
\begin{gathered}
N_{\tau}(k, T)=\sum_{x} N_{\tau}(x, T) e^{i k \cdot x}, k \in[-\pi, \pi]^{d}, \\
N_{\tau}(k, z)=\sum_{T=0}^{\infty} N_{\tau}(k, T) z^{T}, z \in \mathbb{C} .
\end{gathered}
$$

The expectation value for a $T$ step walk is defined by

$$
\langle\cdot\rangle_{\tau}=\sum_{x} \cdot N_{\tau}(x, T) / \sum_{x} N_{\tau}(x, T) .
$$

The mean square displacement $\left\langle\omega(T)^{2}\right\rangle_{\tau}$ of a $T$ step walk is given by

$$
\left\langle\omega(T)^{2}\right\rangle_{\tau}=\left.\frac{-\nabla_{k}^{2} N_{\tau}(k, T)}{N_{\tau}(k, T)}\right|_{k=0}
$$

where $\nabla_{k}^{2}$ is the Laplacian with respect to the variable $k$. 
Let

$$
D(k)=\frac{1}{d} \sum_{j=1}^{d} \cos k_{j}
$$

where the $k_{j}$ 's are the components of $k$. For simple random walk it is well known that

and hence

$$
N_{0}(k, T)=D(k)^{T}
$$

$$
N_{0}(k, z)=(1-z D(k))^{-1} .
$$

We define $\Pi_{\tau}(k, z)$ and $F_{\tau}(k, z)$ by

$$
N_{\tau}(k, z)=\left(1-z D(k)-\Pi_{\tau}(k, z)\right)^{-1}=F_{\tau}(k, z)^{-1} .
$$

The quantity $\Pi_{\tau}(k, z)$ will be used as a measure of the deviation of the self-avoiding walk from the simple walk. The lace expansion is an expansion for $\Pi_{\tau}(k, z)$ that can be used to estimate $\Pi_{\tau}$ and its derivatives.

Denote by $r_{\tau}(k)$ the radius of convergence of the power series (1.4) and let $r_{\tau}=r_{\tau}(0)$. Since $1+U_{s t} \leqq 1$ and $\left|N_{\tau}(k, T)\right| \leqq N_{\tau}(k=0, T)$,

$$
r_{\tau}(k) \geqq r_{\tau} \geqq r_{0}=1 \text {. }
$$

Let

$$
D_{\tau}(a)=\left\{z:|z| \leqq r_{\tau}\left(1+a \tau^{-1} \ln \tau\right)\right\} .
$$

We now outline the main ideas involved in the proof of the main result:

Theorem 1.1. There is a constant $d_{0} \geqq 5$ such that for $d \geqq d_{0}$,

$$
\left\langle\omega(T)^{2}\right\rangle_{T}=D T+O\left(T^{1 / 2} \ln T\right) \text { as } T \rightarrow \infty,
$$

with $D>1$.

The first step in the proof of Theorem 1.1 is to use the lace expansion as in [3] to obtain

where

$$
\left|\Pi_{\tau}(k, z)\right| \leqq\left\|N_{\tau}^{(1)}(x,|z|)\right\|_{\infty}\left[|z|+\sum_{N=2}^{\infty}\left\|N_{\tau}^{(1)}(x,|z|)\right\|_{2}^{N}\left\|N_{\tau}^{(0)}(x,|z|)\right\|_{2}^{2 N-2}\right],
$$

$$
N_{\tau}^{(\alpha)}(x, z)=\sum_{T=\delta_{\alpha, 1}}^{\tau} N_{\tau}(x, T) z^{T},
$$

and the norms are $x$-space $L^{p}$ norms. Similar bounds are obtained for $\partial_{k}^{u} \partial_{z}^{v} \Pi_{\tau}(k, z)$, involving $\left\|x^{u^{\prime}} \partial_{z}^{v^{\prime}} N_{\tau}^{(\alpha)}(x,|z|)\right\|$ with $u^{\prime} \leqq u, v^{\prime} \leqq v$, although if $u \neq 0$ the first term $|z|$ in the square brackets in (1.8) is absent. We then note that the right side of (1.8) should be small, in fact $O\left(d^{-1}\right)$, because the factor $(2 d)^{-T}$ in $N_{\tau}(x, T)$ should make $\left\|N_{\tau}^{(1)}(x,|z|)\right\|_{\infty} \leqq O\left(d^{-1}\right),\left\|N_{\tau}^{(0)}(x,|z|)\right\|_{2} \leqq O(1)$, and $\left\|N_{\tau}^{(1)}(x,|z|)\right\|_{2} \leqq O\left(d^{-1 / 2}\right)$ uniformly in $\tau$ and $z \in D_{\tau}(1 / 2)$. This will be explained in more detail below. The $d^{-1 / 2}$ in the $L^{2}$ norm can be understood from the fact that

$$
\left\|N_{\tau}^{(1)}(x,|z|)\right\|_{2}^{2}=\sum_{x} \sum_{S, T=1}^{\tau} N_{\tau}(x, T) N_{\tau}(x, S)|z|^{S+T}>\sum_{x} N_{\tau}(x, 1)^{2}|z|^{2}=(2 d)^{-1}|z|^{2} .
$$

Similarly $\left|\partial_{k_{\imath}}^{2} \Pi_{\tau}(k, z)\right|$ and $\left|\partial_{z} \Pi_{\tau}(k, z)\right|$ will be bounded by inverse powers of $d$, uniformly in $\tau$ and $z \in D_{\tau}(1 / 2)$. 
Given these bounds on $\Pi_{\tau}$ and its derivatives we argue as in [3] that $N_{\tau}(k, z)$ has a simple pole at $r_{\tau}(k)$ for small $k^{2}$, with $r_{\tau}(k) \in D_{\tau}(1 / 4)$, and is otherwise analytic in $D_{\tau}(1 / 2)$. Then $N_{\tau}(k, T)$ is evaluated using the Cauchy Integral Formula to be the sum of the residue of $-N_{\tau}(k, z) z^{-(T+1)}$ at $r_{\tau}(k)$ and a small correction involving an integral around $\partial D_{\tau}(1 / 2)$, yielding

$$
N_{\tau}(k, T) \approx-\left[\partial_{z} F_{\tau}\left(k, r_{\tau}(k)\right)\right]^{-1} r_{\tau}(k)^{-(T+1)} .
$$

Estimates on $\Pi_{\tau}$ and its derivatives can then be used to show that the dominant contribution to $\nabla_{k}^{2} N_{\tau}(0, T)$ is given by

$$
\nabla_{k}^{2} N_{\tau}(0, T) \approx\left[\partial_{z} F_{\tau}\left(0, r_{\tau}\right)\right]^{-1}(T+1) r_{\tau}^{-(T+2)} \nabla_{k}^{2} r_{\tau}(0) \text {. }
$$

Taking the memory to be $T$ and using (1.9) and (1.10) in (1.5) gives $\left\langle\omega(T)^{2}\right\rangle_{T} \sim D_{T} T$, where $D_{T}=r_{T}^{-1} \nabla_{k}^{2} r_{T}(0)$. It can then be shown that $D_{T}=D+O\left(T^{-1}\right)$ with $D>1$.

We now describe the method for obtaining bounds on norms of $x^{u} \partial_{z}^{v} N_{\tau}^{(1)}(k, z)$, uniformly in $\tau$ and $z \in D_{\tau}(1 / 2)$. First we obtain bounds for $v \leqq 2,|u| \leqq 2,2 v+|u| \leqq 4$, uniformly in $\tau$ and $z \in D_{\tau}(0)$. It is then straightforward to extend the estimates to $z \in D_{\tau}(1 / 2)$ at the expense of one $z$-derivative, i.e., for $v \leqq 1,|u| \leqq 2,2 v+|u| \leqq 2$; see Theorem 4.3. The bounds for $z \in D_{\tau}(0)$ are the main technical problem faced in this paper. This is also the place where our method differs from that of [3].

To obtain the estimates for $z \in D_{\tau}(0)$ we proceed as follows. We first show that for fixed $u, v, \tau$ the relevant norms of $x^{u} \partial_{z}^{v} N_{\tau}^{(\alpha)}(x, \varrho)$ are continuous in $\varrho$. We then show that there are constants $K_{0}$ and $d_{0}$ such that for $d \geqq d_{0}, \varrho \in\left[0, r_{\tau}\right]$, and all $\tau$, $P_{4} \Rightarrow P_{2}$, where $P_{a}$ is the statement that the various norms are bounded above by $a K_{0} d^{-p}$. Here $p$ is the power appropriate to a particular norm and is determined by looking at the leading behaviour of the corresponding simple random walk norm. It then follows from the value of the norms at $\varrho=0$ that they are in fact-bounded above by $2 K_{0} d^{-p}$. This type of argument has been used in a different context in [2]. The basic idea in proving the implication $P_{4} \Rightarrow P_{2}$ will now be illustrated for $\left\|N_{\tau}^{(1)}(x, \varrho)\right\|_{2}$.

Using the assumed bounds $4 K_{0} d^{-p}$ on the norms, it follows from (1.8) that $\left|\Pi_{\tau}(k, z)\right| \leqq K_{1} d^{-1}$, and from the analogue of $(1.8)$ for $\partial_{k_{\imath} k_{j}}^{2} \Pi_{\tau}(k, z)$ that $\left|\partial_{k_{i} k_{j}}^{2} \Pi_{\tau}(k, z)\right|$ $\leqq K_{1}\left(\delta_{i j} d^{-5 / 2}+d^{-3}\right)$, where $K_{1}$ is a constant depending on $K_{0}$. It is only $\varrho \in\left(1, r_{\tau}\right]$ that poses any difficulty, and an elementary argument shows that for $d \geqq d_{0}\left(K_{0}\right)$ and $\varrho \in\left(1, r_{\tau}\right]$,

$$
F_{\tau}(k, \varrho)=F_{\tau}(0, \varrho)+\varrho(1-D(k))+\Pi_{\tau}(0, \varrho)-\Pi_{\tau}(k, \varrho) \geqq c(1-D(k))=c F_{0}(k, 1),
$$

where $c$ is a universal constant which does not depend on $K_{0}$. Then using Parseval's equality to convert an $x$-space $L^{2}$ norm to a $k$-space $L^{2}$ norm gives

$$
\begin{aligned}
&\left\|N_{\tau}^{(1)}(x, \varrho)\right\|_{2} \leqq \\
& \sum_{T=1}^{\infty} N_{\tau}(x, T) \varrho^{T}\left\|_{2}=\right\| N_{\tau}(x, \varrho)-\delta_{x, 0} \|_{2} \\
&=\left\|N_{\tau}(k, \varrho)-1\right\|_{2}=\left\|\frac{\varrho D(k)+\Pi_{\tau}(k, \varrho)}{F_{\tau}(k, \varrho)}\right\|_{2} \\
& \leqq \varrho c^{-1}\left\|\frac{D}{1-D}\right\|_{2}+K_{1} d^{-1} c^{-1}\left\|\frac{1}{1-D}\right\|_{2}
\end{aligned}
$$


The norms on the right side of (1.11) are norms of simple random walk quantities and in $x$-space are respectively $\left\|\sum_{T=1}^{\infty} N_{0}(x, T)\right\|_{2}$ and $\left\|\sum_{T=0}^{\infty} N_{0}(x, T)\right\|_{2}$. These are bounded above by $c_{1} d^{-1 / 2}$ and $c_{1}$ respectively, so

$$
\left\|N_{\tau}^{(1)}(x, \varrho)\right\|_{2} \leqq \varrho c_{1} c^{-1} d^{-1 / 2}+K_{1} c^{-1} c_{1} d^{-1} .
$$

The assumption $P_{4}$ can be used to show that $\varrho \leqq 1+K_{1} d^{-1}$. Thus taking $K_{0} \geqq c_{1} c^{-1}$ and $d$ sufficiently large (depending on $K_{0}$ ) gives $\left\|N_{\tau}^{(1)}(x, \varrho)\right\|_{2}$ $\leqq 2 K_{0} d^{-1 / 2}$. The other norms are handled similarly.

This paper is organized as follows. In the next section the lace expansion is derived and it is shown how to obtain bounds like (1.8). In Sect. 3 estimates are obtained for the various simple random walk norms which are needed as explained above. Section 4 is concerned with convergence of the lace expansion and contains the proof of the implication $P_{4} \Rightarrow P_{2}$ and estimates for $\Pi_{\tau}$ and its derivatives. Finally in Sect. 5 the bounds on $\Pi_{\tau}$ and its derivatives are used to fill in the details of the argument involving the Cauchy Integral Formula sketched above and to complete the proof of Theorem 1.1. The proof that $D$ is greater than one can be found at the end of Sect. 5 .

\section{The Lace Expansion}

This section contains a derivation of the lace expansion, following $[3,1]$.

Elements of the set $\mathscr{B}_{\tau}(I)$ defined in (1.1) are referred to as bonds. Define

Then

$$
\psi(I)=\prod_{s t \in \mathscr{B}_{\tau}(I)}\left(1+U_{s t}\right)
$$

$$
\psi(I)=\sum_{B \subset \mathscr{B}_{\tau}(I)} \prod_{s t \in B} U_{s t}
$$

A connected graph $G$ on $I$ is defined to be a subset of $\mathscr{B}_{\tau}(I)$ such that each endpoint of $I$ is part of a bond in $G$, and for each $m$ in the interior of $I$ there is a bond $s t \in G$ with $m \in(s, t)$. Subsets of $\mathscr{B}_{\tau}(I)$ are in a one-one correspondence with partitions of $I$ into ordered subintervals $I_{1}, \ldots, I_{n}$ with disjoint interiors but possibly overlapping endpoints, with a connected graph on each $I_{j}$, as in Fig. 2.1. A subinterval may consist of a single point. It follows that

$$
\psi(I)=\sum_{n} \sum_{I_{1}, \ldots, I_{n}} \psi_{c}\left(I_{1}\right) \ldots \psi_{c}\left(I_{n}\right)
$$

where the sum is over partitions of $I$ as above and

$$
\psi_{c}(I)=\sum_{G} \prod_{s t \in G} U_{s t}
$$

the sum being over connected graphs $G$ on $I$. We use the convention that if $I$ consists of a single point then $\psi_{c}(I)=1$.

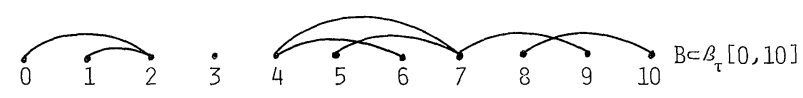

Fig. 2.1

$$
I_{1}=[0,2], I_{2}=[3,3], I_{3}=[4,7], I_{4}=[7,10]
$$


Fig. 2.2
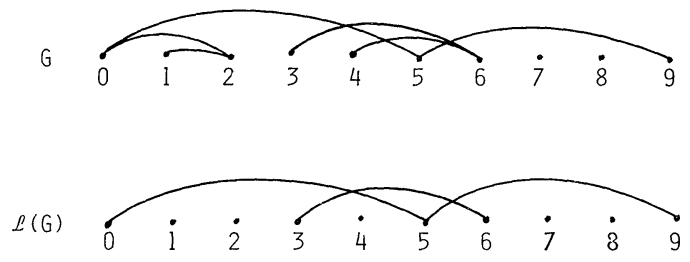

Let $\mathscr{L}_{\tau}(I)$ denote the set of connected graphs on $I$ such that the removal of any one bond from the graph results in a graph which is not connected. Elements of $\mathscr{L}_{\tau}(I)$ are called laces. The following prescription defines a way of obtaining from any connected graph $G \subset \mathscr{B}_{\tau}[a, b]$ a lace $\mathscr{L}(G) \subset G . \mathscr{L}(G)$ has bonds $s_{1} t_{1}, s_{2} t_{2}, \ldots$, where

$$
\begin{gathered}
s_{1}=a, \quad t_{1}=\max \{t: a t \in G\}, \\
t_{i+1}=\max \left\{t: s t \in G, s<t_{i}\right\}, \\
s_{i}=\min \left\{s: s t_{i} \in G\right\} .
\end{gathered}
$$

An example is shown in Fig. 2.2. Given a lace $L$, the set of all bonds $s t \in \mathscr{B}_{\tau}(I) \backslash L$ such that $\mathscr{L}(L \cup\{s t\})=L$ is denoted by $\mathscr{C}_{\tau}(L)$. Bonds in $\mathscr{C}_{\tau}(L)$ are said to be compatible with $L$.

With these definitions we have

$$
\begin{aligned}
\psi_{c}(I) & =\sum_{G \text { on } I} \prod_{s t \in G} U_{s t}=\sum_{L \in \mathscr{L}_{\tau}(I)} \prod_{s t \in L} U_{s t} \sum_{G: \mathscr{L}(G)=L} \prod_{s t \in G \backslash L} U_{s t} \\
& =\sum_{L \in \mathscr{L}_{\tau}(I)} \prod_{s t \in L} U_{s t} \prod_{s t \in \mathscr{C}_{\tau}(L)}\left(1+U_{s t}\right) .
\end{aligned}
$$

The following theorem gives the lace expansion for $\Pi_{\tau}(k, z)$.

Theorem 2.1 (Brydges-Spencer).

$$
\Pi_{\tau}(k, z)=\sum_{T=1}^{\infty} \sum_{\omega,|\omega|=T}\left(\frac{z}{2 d}\right)^{T} e^{i k \omega(T)} \sum_{L \in \mathscr{L}_{\tau}[0, T]} \prod_{s t \in L} U_{s t} \prod_{s t \in \mathscr{C}_{\tau}(L)}\left(1+U_{s t}\right) .
$$

Proof. By Eqs. (1.2-4), (2.1), and (2.3),

$$
\begin{aligned}
N_{\tau}(k, z) & =\sum_{T=0}^{\infty}\left(\frac{z}{2 d}\right)^{T} \sum_{\omega,|\omega|=T} e^{i k \omega(T)} \psi([0, T]) \\
& =1+\sum_{T=1}^{\infty}\left(\frac{z}{2 d}\right)^{T} \sum_{\omega,|\omega|=T} e^{i k \omega(T)} \sum_{n=1}^{T+1} \sum_{I_{1}, \ldots, I_{n}} \psi_{c}\left(I_{1}\right) \ldots \psi_{c}\left(I_{n}\right) .
\end{aligned}
$$

The contribution to the sum on the right side coming from partitions with $I_{1}=[0,0]$ is

$$
\begin{aligned}
& \sum_{T=1}^{\infty}\left(\frac{z}{2 d}\right)^{T} \sum_{\omega,|\omega|=T} e^{i k \omega(T)} \sum_{n=2}^{T+1} \sum_{I_{2}, \ldots, I_{n}} \psi_{c}\left(I_{2}\right) \ldots \psi_{c}\left(I_{n}\right) \\
& \quad=\sum_{T=1}^{\infty}\left(\frac{z}{2 d}\right)^{T} \sum_{\omega,|\omega|=T} e^{i k \omega(T)} \psi([1, T]) \\
& \quad=\sum_{T=1}^{\infty}\left(\frac{z}{2 d}\right)^{T-1} \sum_{\omega,|\omega|=T} e^{i k(\omega(T)-\omega(1))} \psi([1, T]) \frac{z}{2 d} e^{i k \omega(1)}=z D(k) N_{\tau}(k, z) .
\end{aligned}
$$


The contribution to the sum on the right side of (2.7) due to partitions with $I_{1} \neq[0,0]$ is given by the following expression, where $s \geqq 1$ is the upper limit of $I_{1}=[0, s]$ :

$$
\begin{aligned}
\sum_{T}^{\infty} & \left(\frac{z}{2 d}\right)^{T} \sum_{\omega,|\omega|=T} e^{i k \omega(T)} \sum_{s=1}^{T} \psi_{c}([0, s]) \psi([s, T]) \\
& =\sum_{s=1}^{\infty}\left(\frac{z}{2 d}\right)^{s} \sum_{T=s}^{\infty} \sum_{T=|\omega|=T} e^{i k \omega(s)} \psi_{c}([0, s])\left(\frac{z}{2 d}\right)^{T-s} e^{i k(\omega(T)-\omega(s))} \psi([s, T]) \\
& =\sum_{s=1}^{\infty}\left(\frac{z}{2 d}\right)^{s} \sum_{\omega,|\omega|=s} e^{i k \omega(s)} \psi_{c}([0, s]) N_{\tau}(k, z) .
\end{aligned}
$$

Replacing the sum on the right side of Eq. (2.7) by (2.8) and (2.9), using (2.5), and comparing with the definition of $\Pi_{\tau}(k, z)$ in Eq. (1.7) completes the proof.

The quantity $\prod_{s t \in L} U_{s t}$ in the right side of (2.6) gives a nonzero contribution to $\Pi_{\tau}(k, z)$ only for walks which intersect themselves as indicated in Fig. 2.3. The product over $\mathscr{C}_{\tau}(L)$ in (2.6) disallows many but in general not all other selfintersections. The generic walk whose topology is that corresponding to a lace with $N$ bonds will be denoted $G_{N}$. Consider the walk $G_{N}$ to consist of $2 N-1$ subwalks over time intervals $\left[0, s_{2}\right],\left[s_{2}, t_{1}\right],\left[t_{1}, s_{3}\right],\left[s_{3}, t_{2}\right], \ldots,\left[t_{N-1}, T\right]$. Each subwalk consists of not more than $\tau$ steps because every bond st in a lace $L \in \mathscr{L}_{\tau}[0, T]$ satisfies $|s-t| \leqq \tau$. Also, it is consistent with the definition of a lace to have $t_{i}=s_{i+2}$ for $i \geqq 1$ but inconsistent to have $t_{i}=s_{i+1}$ for $i \geqq 1$, and so for $N \geqq 2$ at least $N+1$ of the subwalks consist of at least one step. Except for $G_{1}$, no subwalk consisting of at least one step on an interval $[\alpha, \beta]$ begins and ends at the same place since $\alpha \beta \in \mathscr{C}_{\tau}(L)$. The set of lines in $G_{N}$ which must consist of at least one step is denoted by $G_{N}^{(1)}$. The remaining lines, which may have zero length, comprise the set $G_{N}^{(0)}$. In Fig. 2.3 lines in $G_{N}^{(0)}$ are slashed.

The lace expansion (2.6) can be used to obtain an upper bound on $\left|\Pi_{\tau}(k, z)\right|$ as follows: take absolute values inside the sums of (2.6), factor $\left(\frac{|z|}{2 d}\right)^{T}$ among the
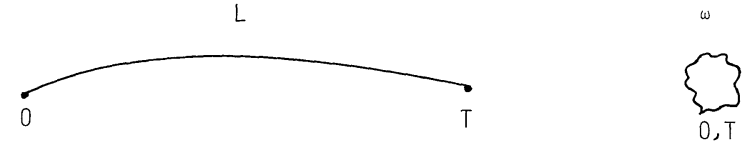

$G_{1}$
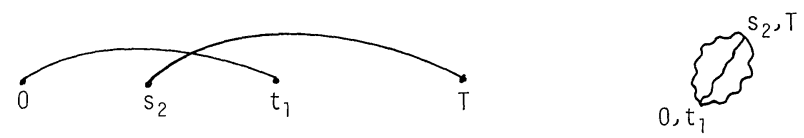

$G_{2}$
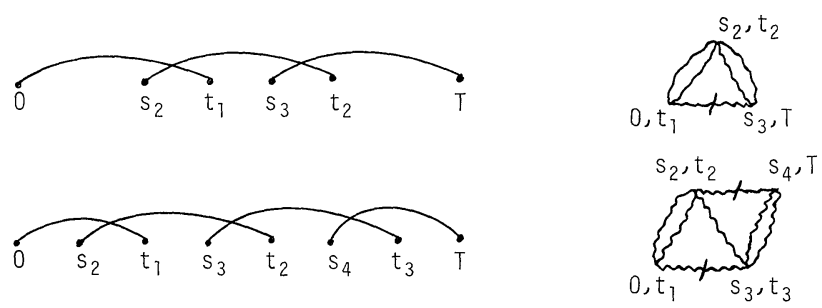

$G_{3}$

$G_{4}$

Fig. 2.3

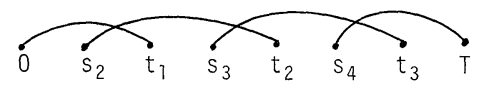


subwalks, and omit factors $\left(1+U_{s t}\right)$ whenever st is a bond linking two distinct subwalks. We let $x_{1}=0$ and denote the position of a generic walk at time $s_{i}$ by $x_{2 i-2}, i \geqq 2$ and at time $t_{i}$ by $x_{2 i+1}, i \geqq 1$. (Many $x_{i}$ 's are equal, for example for $G_{3}$ $x_{3}=x_{1}=0, x_{5}=x_{2}$, and $x_{6}=x_{4}$.) We denote the line in $G_{N}$ corresponding to the subwalk from $x_{i}$ to $x_{i+1}$ by $\ell_{i}$. With this notation the upper bound is

$$
\begin{aligned}
\left|\Pi_{\tau}(k, z)\right| \leqq & \sum_{T=2}^{\tau} \sum_{\omega:|\omega|=T}\left(\frac{|z|}{2 d}\right)^{T}\left|U_{0 T}\right| \prod_{s t \in \mathscr{C}_{\tau}(L=0 T)}\left(1+U_{s t}\right) \\
& +\sum_{N=2}^{\infty} \sum_{x_{2}, \ldots, x_{2 N} \in \mathbb{Z}^{d}} \prod_{\alpha=0}^{1} \prod_{\left\{i: \ell_{\imath} \in G_{N}^{(\alpha)}\right\}} N_{\tau}^{(\alpha)}\left(x_{i+1}-x_{i},|z|\right),
\end{aligned}
$$

where

$$
N_{\tau}^{(\alpha)}(x, z)=\sum_{T=\delta_{\alpha, 1}}^{\tau} N_{\tau}(x, T) z^{T} .
$$

The first term on the right side of (2.9) is special in that it cannot be bounded above by $N_{\tau}^{(1)}(0,|z|)=0$. However using $\langle x, 0\rangle$ to indicate the nearest neighbours of the origin we have

$$
\begin{aligned}
\sum_{T=2}^{\tau} \sum_{\omega,|\omega|=T}\left(\frac{|z|}{2 d}\right)^{T}\left|U_{0 T}\right| \prod_{s t \in \mathscr{C}_{\tau}(0 T)}\left(1+U_{s t}\right) & =\frac{|z|}{2 d} \sum_{\langle x, 0\rangle} \sum_{T=2}^{\tau} N_{\tau}(x, T-1)|z|^{T-1} \\
& \leqq|z|\left\|N_{\tau}^{(1)}(x,|z|)\right\|_{\infty},
\end{aligned}
$$

where the norm is the $x$-space $L^{\infty}$ norm. It is shown in [3] how to use the Young and Hölder inequalities to obtain the bound

$$
\begin{aligned}
& \sum_{x_{2}, \ldots, x_{2 N}} \prod_{\alpha=0}^{1} \prod_{\left\{i: \ell_{\imath} \in G_{N}^{(\alpha)}\right\}} N_{\tau}^{(\alpha)}\left(x_{i+1}-x_{i},|z|\right) \\
& \quad \leqq\left\|N_{\tau}^{(1)}(x,|z|)\right\|_{\infty}\left\|N_{\tau}^{(1)}(x,|z|)\right\|_{2}^{N}\left\|N_{\tau}^{(0)}(x,|z|)\right\|_{2}^{N-2} .
\end{aligned}
$$

The norms here are $x$-space $L^{p}$ norms. Thus from (2.9) we have

$$
\left|\Pi_{\tau}(k, z)\right| \leqq\left\|N_{\tau}^{(1)}(x,|z|)\right\|_{\infty}\left[|z|+\sum_{N=2}^{\infty}\left\|N_{\tau}^{(1)}(x,|z|)\right\|_{2}^{N}\left\|N_{\tau}^{(0)}(x,|z|)\right\|_{2}^{N-2}\right] .
$$

Similarly upper bounds on $k$ and $z$ derivatives of $\Pi_{\tau}$ can be obtained by factoring both $\left(\frac{|z|}{2 d}\right)^{T}$ and $e^{i k \cdot \omega(T)}$ among the subwalks and using the product rule to have the derivatives act on single subwalks, giving

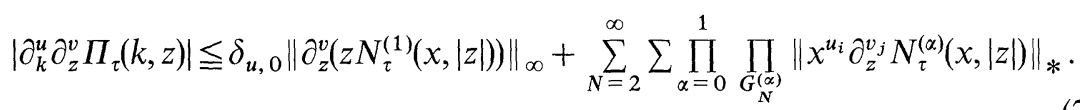

In the first term on the right side the derivative is performed before evaluating $N_{\tau}^{(1)}$ at $|z|$. For $u \neq 0$ the first term on the right side is absent because for $G_{1}, \omega(T)=0$, and so the contribution to $\Pi_{\tau}$ from $G_{1}$ is independent of $k$. The unlabelled sum is over ways of choosing nonnegative multi-indices $u_{i}$ such that $\Sigma u_{i}=u$ and nonnegative $v_{j}$ 
such that $\Sigma v_{j}=v$. Any one norm in the product can be taken to be the $L^{\infty}$ norm. The others are $L^{2}$ norms. The unlabelled sum consists of $(2 N-1)^{|u|+v}$ terms.

Since the contribution from $G_{1}$ to $\Pi_{\tau}(k, z)$ is independent of $k$,

$$
\left|\Pi_{\tau}(k, z)-\Pi_{\tau}(0, z)\right| \leqq 2\left\|N_{\tau}^{(1)}(x,|z|)\right\|_{\infty} \sum_{N=2}^{\infty}\left\|N_{\tau}^{(1)}(x,|z|)\right\|_{2}^{N}\left\|N_{\tau}^{(0)}(x,|z|)\right\|_{2}^{N-2}
$$

To control the rate of convergence of the diffusion constant $D_{\tau}$ to its limiting value $D$ it will be necessary to have estimates for $\delta \Pi(k, z)=\Pi_{\tau}(k, z)-\Pi_{\sigma}(k, z)$ and its derivatives. For $\tau>\sigma, \mathscr{L}_{\sigma}[0, T] \subset \mathscr{L}_{\tau}[0, T]$ and for $L \in \mathscr{L}_{\sigma}[0, T], \mathscr{C}_{\sigma}(L) \subset \mathscr{C}_{\tau}(L)$. This last inclusion is often strict, but if $L$ contains no bond of length greater than $\frac{\sigma}{2}$, then $\mathscr{C}_{\tau}(L)$ can contain no bond of length greater than $\sigma$, and hence $\mathscr{C}_{\sigma}(L)=\mathscr{C}_{\tau}(L)$. Therefore in $\delta \Pi$ there is a cancellation of all terms involving laces with all bonds of length less than or equal to $\frac{\sigma}{2}$ and

$$
|\delta \Pi(k, z)| \leqq\left|\Pi_{\tau}^{\prime}(k, z)\right|+\left|\Pi_{\sigma}^{\prime}(k, z)\right|
$$

where $\Pi^{\prime}$ is defined by the right side of (2.6) with just laces having at least one bond of length greater than $\frac{\sigma}{2}$ participating.

At least one of the subwalks corresponding to a lace with a bond of length greater than $\frac{\sigma}{2}$ must consist of $\frac{\sigma}{6}$ or more steps. By the same argument used to derive (2.12) we have

$$
\begin{aligned}
& \left|\partial_{k}^{u} \partial_{z}^{v} \delta \Pi(k, z)\right| \leqq 2\left[\delta_{u, 0}\left\|\partial_{z}^{v}(z \delta N(x,|z|))\right\|_{\infty}\right. \\
& \left.\quad+\sum_{N=2}^{\infty} \sum \prod_{\alpha=0}^{1} \prod_{G_{N}^{(\alpha)}}\left\|x^{u_{i}} \partial_{z}^{v_{j}} \delta^{*} N^{(\alpha)}(x,|z|)\right\|_{*}\right] .
\end{aligned}
$$

Here

$$
\delta N(x,|z|)=\max \left\{\sum_{T=\sigma / 6}^{\sigma} N_{\sigma}(x, T)|z|^{T}, \sum_{T=\sigma / 6}^{\tau} N_{\tau}(x, T)|z|^{T}\right\},
$$

one $\delta^{*} N^{(\alpha)}$ is chosen to be $\delta N$, and the remainder are taken to be $\max \left\{N_{\sigma}^{(\alpha)}, N_{\tau}^{(\alpha)}\right\}$. The unlabelled sum also extends over ways of assigning one $\delta^{*} N^{(\alpha)}$ to be $\delta N$. One norm in the product is an $L^{\infty}$ norm and the remainder are $L^{2}$ norms.

\section{Estimates for Simple Random Walk}

The proof of convergence of (2.12-14) will be obtained in Sect. 4 using estimates for simple random walk which we obtain in this section. 
By virtue of its definition in Eq. (1.2), $N_{0}(x, T)$ is the probability that a $T$ step simple random walk starting at the origin ends at $x$. Since $D(k)$ [defined in (1.6)] is the characteristic function of a single step,

$$
N_{0}(x, 2 T+S)=(2 \pi)^{-d} \int d k e^{-i k x} D(k)^{2 T+S} \leqq(2 \pi)^{-d} \int d k D(k)^{2 T}=N_{0}(0,2 T) .
$$

Here we have used the fact that $|D(k)| \leqq 1$. The integrals extend over $[-\pi, \pi]^{d}$, and $S, T=0,1,2, \ldots$ Also, since a $2 T$ step walk which ends at the origin must lie in a $T$ dimensional subspace of $\mathbb{Z}^{d}$, for $T \leqq d$ we have

$$
N_{0}(0,2 T) \leqq\left(\begin{array}{l}
d \\
T
\end{array}\right)\left(\frac{T}{d}\right)^{2 T} \leqq \frac{T^{2 T}}{T ! d^{T}}
$$

The following lemma is a simple extension of a result of [5]. In the proof $c$ stands for a universal constant which may be different in different occurrences.

\section{Lemma 3.1.}

$$
\sum_{T=1}^{\infty} T^{3} N_{0}(0,2 T) \leqq O\left(d^{-1}\right)
$$

Proof. The sum of the first four terms on the left side of (3.3) is $O\left(d^{-1}\right)$ by (3.2). By (3.1) and (3.2),

$$
\sum_{T=5}^{d-1} T^{3} N_{0}(0,2 T) \leqq \sum_{T=5}^{d-1} T^{3} N_{0}(0,10) \leqq(d-1)^{3}(d-5) \frac{5^{10}}{5 ! d^{5}} \leqq O\left(d^{-1}\right) .
$$

To bound the sum over $T \geqq d$ we observe that

$$
\begin{aligned}
& \sum_{T=d}^{\infty} T^{3} N_{0}(0,2 T)<\sum_{T=d}^{\infty}(2 T)(2 T-1)(2 T-2)(2 \pi)^{-d} \int D(k)^{2 T} d k \\
& \quad=(2 \pi)^{-d} \int\left[\sum_{m=2 d}^{\infty} m(m-1)(m-2) D(k)^{m-3}\right] D(k)^{3} d k .
\end{aligned}
$$

The factor in square brackets is the third derivative of $x^{2 d}(1-x)^{-1}$ evaluated at $x=D(k)$. Explicit evaluation of this derivative together with $|D(k)| \leqq 1$ can be used to bound (3.4) above by

$$
c d^{3}(2 \pi)^{-d} \int d k D(k)^{2 d} \sum_{p=1}^{4}(1-D(k))^{-p} .
$$

Arguing as in [5] we observe that if $0 \leqq k_{j} \leqq \frac{\pi}{2} \leqq k_{l} \leqq \pi$ for $j=1, \ldots, m$ and $l=m+1, \ldots, d$, then

$$
\begin{aligned}
|D(k)| & \leqq d^{-1} \sum_{i=1}^{d}\left|\cos k_{i}\right| \leqq 1-4 \pi^{-2} d^{-1} \sum_{j=1}^{m} k_{j}^{2}-4 \pi^{-2} d^{-1} \sum_{l=m+1}^{d}\left(\pi-k_{l}\right)^{2} \\
& \leqq \exp \left[-4 \pi^{-2} d^{-1}\left(\sum_{j=1}^{m} k_{j}^{2}+\sum_{l=m+1}^{d}\left(\pi-k_{l}\right)^{2}\right)\right] .
\end{aligned}
$$


By symmetry and (3.5),

$$
\begin{aligned}
& d^{3}(2 \pi)^{-d} \int D(k)^{2 d}(1-D(k))^{-p} d k \\
&=d^{3} \pi^{-d} \sum_{m=0}^{d}\left(\begin{array}{c}
d \\
m
\end{array}\right) \int_{0}^{\pi / 2} d k_{1} \ldots d k_{m} \int_{\pi / 2}^{\pi} d k_{m+1} \ldots d k_{d} D(k)^{2 d}(1-D(k))^{-p} \\
& \leqq d^{3}\left(\frac{2}{\pi}\right)^{d} \int_{\left[0, \frac{\pi}{2}\right]^{d}} d k \exp \left(-8 \pi^{-2} k^{2}\right)\left(4 \pi^{-2} \mathrm{~d}^{-1} k^{2}\right)^{-p} \\
& \leqq d^{3} \pi^{-d}\left(\frac{\pi^{2} d}{4}\right)^{p} \int_{(-\infty, \infty)^{d}} d k k^{-2 p} \exp \left(-8 \pi^{-2} k^{2}\right) \\
&= d^{3} \pi^{-d}\left(\frac{\pi^{2} d}{4}\right)^{p} \frac{2 \pi^{d / 2}}{\Gamma(d / 2)} \int_{0}^{\infty} d t t^{d-2 p-1} \exp \left(-8 \pi^{-2} t^{2}\right) \\
&= d^{3}\left(\frac{\pi^{2} d}{4}\right)^{p} \frac{\pi^{-d / 2}}{\Gamma(d / 2)}\left(\frac{\pi^{2}}{8}\right)^{\frac{d}{2}-p} \Gamma\left(\frac{d}{2}-p\right) \\
&= \frac{c d^{p+3} \Gamma\left(\frac{d}{2}-p\right)}{\Gamma\left(\frac{d}{2}\right)}\left(\frac{\pi}{8}\right)^{d / 2} \leqq O\left(d^{-1}\right) .
\end{aligned}
$$

This provides the required bound for the left side of (3.4) and the proof is complete.

We now use Lemma 3.1 to obtain bounds on $L^{2}$ and $L^{\infty}$ norms of $x^{u} \partial_{z}^{v} \sum_{T=1}^{\infty} N_{0}(x, T) z^{T}$ for $|z|$ less than or equal to the critical value of 1 , where $N_{0}(k=0, z)$ diverges.

\section{Lemma 3.2.}

(a)

$$
\begin{aligned}
& \left\|\partial_{z}^{v} \sum_{T=1}^{\infty} N_{0}(x, T) z^{T}\right\|_{\infty} \leqq O\left(d^{-1}\right), \quad v=0,1,2,|z| \leqq 1 . \\
& \left\|\partial_{z}^{v} \sum_{T=1}^{\infty} N_{0}(x, T) z^{T}\right\|_{2}^{2} \leqq O\left(d^{-1}\right), \quad v=0,1,|z| \leqq 1 .
\end{aligned}
$$

Proof. (a) For $v=0,1,2$ and $|z| \leqq 1$,

$$
\left|\partial_{z}^{v} \sum_{T=1}^{\infty} N_{0}(x, T) z^{T}\right| \leqq \sum_{T=1}^{\infty} T^{2} N_{0}(x, T) .
$$

By (3.1) and Lemma 3.2,

$$
\begin{aligned}
\sum_{T=1}^{\infty} T^{2} N_{0}(x, T)= & N_{0}(x, 1)+\sum_{T=1}^{\infty}(2 T)^{2} N_{0}(x, 2 T) \\
& +\sum_{T=1}^{\infty}(2 T+1)^{2} N_{0}(x, 2 T+1) \leqq \frac{1}{2 \bar{d}}+O\left(d^{-1}\right)
\end{aligned}
$$


(b) For $v=0,1$ and $|z| \leqq 1$,

$$
\begin{aligned}
& \left\|\partial_{z}^{v} \sum_{T=1}^{\infty} N_{0}(x, T) z^{T}\right\|_{2}^{2} \leqq\left\|\sum_{T=1}^{\infty} T N_{0}(x, T)\right\|_{2}^{2}=\sum_{S \cdot T=1}^{\infty} \sum_{x} N_{0}(x, T) N_{0}(x, S) S T \\
& \quad=\sum_{S, T=1}^{\infty} S T N_{0}(0, S+T) \leqq \sum_{n=2}^{\infty}(n-1)^{3} N_{0}(0, n) \leqq O\left(d^{-1}\right),
\end{aligned}
$$

using Lemma 3.1 in the last step.

\section{Lemma 3.3.}

$$
1<\left\|N_{0}(x, z=1)\right\|_{2}^{2} \leqq 1+O\left(d^{-1}\right) .
$$

Proof. Since $N_{0}(x, T=0)=\delta_{x, 0}$,

$$
\left\|N_{0}(x, z=1)\right\|_{2}^{2}=\left\|\delta_{x, 0}+\sum_{T=1}^{\infty} N_{0}(x, T)\right\|_{2}^{2}=1+2 \sum_{T=1}^{\infty} N_{0}(0, T)+\left\|\sum_{T=1}^{\infty} N_{0}(x, T)\right\|_{2}^{2} .
$$

By Lemma 3.2 the sums on the right side are $O\left(d^{-1}\right)$.

Before dealing with norms where a factor $x^{u}$ is present we derive a consequence of Lemma 3.3 for certain $k$-space integrals. It follows from the definition of $D(k)$ that

$$
\frac{2}{\pi^{2} d} k^{2} \leqq 1-D(k) \leqq \frac{1}{2 d} k^{2}
$$

Let $B=\left\{k \in R^{d}:|k|<d^{1 / 2}\right\}$. Then for $2 \leqq m \leqq[(d-1) / 2]$,

$$
\begin{aligned}
(2 \pi)^{-d} \int(1-D)^{-m} d k= & (2 \pi)^{-d} \int_{B}(1-D)^{-m} d k \\
& +(2 \pi)^{-d} \int_{[-\pi, \pi]^{d} \backslash B}(1-D)^{-m} d k \equiv I_{1}+I_{2} .
\end{aligned}
$$

Now by (3.8) and Stirling's formula,

$$
\begin{aligned}
I_{1} & \leqq(2 \pi)^{-d} \frac{2 \pi^{d / 2}}{\Gamma(d / 2)} \int_{0}^{d^{1 / 2}} d t t^{d-1-2 m}\left(\frac{\pi^{2} d}{2}\right)^{m} \\
& \leqq \frac{c(m)}{d-2 m}\left(\frac{1}{2 \sqrt{\pi}}\right)^{d} \frac{d^{d / 2}}{\Gamma(d / 2)} \sim \frac{c(m)}{d-2 m}\left(\frac{e}{2 \pi}\right)^{d / 2} \frac{1}{\sqrt{\pi d}} \leqq O(1)
\end{aligned}
$$

The term $I_{2}$ is bounded as follows:

$$
I_{2} \leqq(2 \pi)^{-d} \int_{[-\pi, \pi]^{d} \backslash B}(1-D)^{-2}\left(\frac{\pi^{2} d}{2 k^{2}}\right)^{m-2} d k \leqq\left(\frac{\pi^{2}}{2}\right)^{m-2}(2 \pi)^{-d} \int_{[-\pi, \pi]^{d}}(1-D)^{-2} d k
$$

By Parseval's equality and Lemma 3.3 the right-hand side of (3.11) is $O(1)$ and hence

$$
(2 \pi)^{-d} \int(1-D)^{-m} d k \leqq O(1), \quad m=2,3,4, \ldots,[(d-1) / 2]
$$


The estimate (3.12) will be used repeatedly. Note that in the next lemma the $L^{2}$ norm is not squared, unlike in Lemma 3.2(b).

Lemma 3.4. For $v=0,1,|z| \leqq 1$ and $p=2$ or $\infty$,

$$
\begin{aligned}
&\left\|x_{i} \partial_{z}^{v} \sum_{T=1}^{\infty} N_{0}(x, T) z^{T}\right\|_{p} \leqq O\left(d^{-1}\right), \\
&\left\|x_{i} x_{j} \partial_{z}^{v} \sum_{T=1}^{\infty} N_{0}(x, T) z^{T}\right\|_{p} \leqq \delta_{i j} O\left(d^{-1}\right)+O\left(d^{-2}\right) .
\end{aligned}
$$

Proof. Part (a) is an immediate consequence of (b) since $\left|x_{i}\right| \leqq x_{i}^{2}$. The left side of (b) is bounded above by putting $v=1$ and $z=1$. Let $q=\frac{p}{p-1}$. Then

$$
\left\|x_{i} x_{j} \partial_{z}^{v} \sum_{T=1}^{\infty} N_{0}(x, T) z^{T}\right\|_{p} \leqq\left\|\partial_{k_{i} k_{j}}^{2} \partial_{z} N_{0}(k, z=1)\right\|_{q} .
$$

Throughout this paper $k$-space norms are with respect to normalized Lebesgue measure on $[-\pi, \pi]^{d}$. Using subscripts to denote partial derivatives,

$$
\begin{aligned}
\partial_{k_{\imath} k_{j}}^{2} \partial_{z} N_{0}(k, 1)= & (1-D)^{-2} D_{i j}+4(1-D)^{-3} D_{i} D_{j}+2(1-D)^{-3} D D_{i j} \\
& +6(1-D)^{-4} D D_{i} D_{j} .
\end{aligned}
$$

By (1.6), $\left|D_{i}\right| \leqq d^{-1}$ and $\left|D_{i j}\right| \leqq \delta_{i j} d^{-1}$. With these inequalities and (3.12) the lemma is proved.

\section{Analyticity of $\Pi$}

In this section we show convergence of $(2.12)$ to prove that $\Pi_{\tau}(k, z)$ and $\partial_{k_{i} k_{j}}^{2} \Pi_{\tau}(k, z)$ are analytic in $z \in D_{\tau}(1 / 2)$ and suitably bounded. First bounds are obtained on $L^{2}$ and $L^{\infty}$ norms of $x^{u} \partial_{z}^{v} N_{\tau}^{(1)}(x, \varrho)$ for $|u| \leqq 2, v \leqq 2,|u|+2 v \leqq 4$, uniformly in $\varrho \in\left[0, r_{\tau}\right]$. Then these bounds are used to bound $L^{2}$ and $L^{\infty}$ norms of $x^{u} \partial_{z}^{v} N_{\tau}^{(1)}(x, \varrho)$ for $|u| \leqq 2$, $v \leqq 1, \quad|u|+2 v \leqq 2$ (i.e., one less $z$-derivative than above) uniformly in $\varrho \in\left[0, r_{\tau}\left(1+(1 / 2) \tau^{-1} \ln \tau\right)\right]$. The method is conceptually simpler than that used in [3] and could be used to give an alternate proof of the results of [3]. However, the extra $z$-derivative used here would lead to divergent integrals in $d=5$ and would have to be replaced by a fractional derivative.

They key idea is contained in the following theorem. It is similar in form to an idea used in [2]. The universal constant $K_{0}$ in part (b) will be fixed in the course of the proof.

Theorem 4.1. (a) For fixed $\tau$ and $d$ and for any $u$ and $v$, the norms $\left\|x^{u} \partial_{z}^{v} N_{\tau}^{(1)}(x, \varrho)\right\|_{2, \infty}$ are continuous in $\varrho \in \mathbb{R}$.

(b) There is a universal constant $d_{0}$ such that for $d \geqq d_{0}, \varrho \in\left[0, r_{\tau}\right]$ and all $\tau$, $P_{4} \Rightarrow P_{2}$, where $P_{a}$ is the following:

$$
P_{a}: \begin{cases}\left\|x^{u} \partial_{z}^{v} N_{\tau}^{(1)}(x, \varrho)\right\|_{2}^{1+\delta_{u, 0}} \leqq a K_{0} d^{-s}, & |u| \leqq 2, v \leqq 1 \\ \left\|\partial_{z}^{v} N_{\tau}^{(1)}(x, \varrho)\right\|_{\infty} \leqq a K_{0} d^{-1}, & v \leqq 2\end{cases}
$$

Here $s=1$ unless $x^{u}=x_{i} x_{j}$ with $i \neq j$ in which case $s=2$. 
Corollary 4.2. (a) With parameters fixed as in Theorem 4.1 (b),

Also

$$
\left\|x^{u} \partial_{z}^{v} N_{\tau}^{(1)}(x, \varrho)\right\|_{2}^{1+\delta_{u, 0}} \leqq 2 K_{0} d^{-s} \quad \text { and } \quad\left\|\partial_{z}^{v} N_{\tau}^{(1)}(x, \varrho)\right\|_{\infty} \leqq 2 K_{0} d^{-1} \text {. }
$$

$$
\left\|x^{u} \partial_{z}^{v} N_{\tau}^{(0)}(x, \varrho)\right\|_{2}^{1+\delta_{u, 0}} \leqq 2 \text { and }\left\|\partial_{z}^{v} N_{\tau}^{(0)}(x, \varrho)\right\|_{\infty} \leqq 2 .
$$

(b) For $|u| \leqq 2$ and $v \leqq 2$ with $|u|+2 v \leqq 4, \partial_{k}^{u} \Pi_{\tau}(k, z)$ is analytic in $D_{\tau}(0)$ with

$$
\left|\partial_{k}^{u} \partial_{z}^{v} \Pi_{\tau}(k, z)\right| \leqq \delta_{u, 0} O\left(d^{-1}\right)+O\left(d^{-5 / 2}\right)
$$

uniformly in $\tau$ and $z \in D_{\tau}(0)$.

Proof of Corollary 4.2. (a) For $\varrho=0 P_{2}$ is satisfied, and hence by Theorem $4.1 P_{2}$ holds for $\varrho \in\left[0, r_{\tau}\right]$. This proves the inequalities involving $N_{\tau}^{(1)}$. The inequalities involving $N_{\tau}^{(0)}$ then follow from the fact that $N_{\tau}^{(0)}(x, \varrho)=\delta_{x, 0}+N_{\tau}^{(1)}(x, \varrho)$.

(b) The desired bound on the derivatives of $\Pi_{\tau}$ follows from part (a) and (2.12), where in the sum over $N$ the $L^{\infty}$ norm is always associated with factors having no $k$-derivatives. The factor $z$ in the right side of $(2.12)$ is bounded because $1-\varrho$ $-\Pi_{\tau}(0, \varrho) \geqq 0$ for $\varrho \leqq r_{\tau}$ by definition of $r_{\tau}$, and therefore by $(2.12) \varrho-1 \leqq \varrho$ const $d^{-1}$. It follows that $\varrho \leqq 1+O\left(d^{-1}\right)$.

The proof of Theorem 4.1 (b) begins by using (2.12) to convert the assumed bounds $P_{4}$ into bounds on $\partial_{k}^{u} \partial_{z}^{v} \Pi_{\tau}(k, \varrho)$. These bounds are then used to show that there is a constant $c$ such that $1-\varrho D(k)-\Pi_{\tau}(k, \varrho) \geqq c(1-D(k))$. The $x$-space norms of $x^{u} \partial_{z}^{v} N_{\tau}^{(1)}(x, \varrho)$ are bounded by $k$-space norms of $\partial_{k}^{u} \partial_{z}^{v} N_{\tau}(k, \varrho)$, which are in turn bounded by corresponding simple random walk norms using the above inequality. The simple random walk norms were controlled in Sect. 3. Any contributions coming from $\partial_{k}^{u} \partial_{z}^{v} \Pi_{\tau}(k, \varrho)$ are multiplied by an inverse power of $d$ which compensates for any coefficients $4 K_{0}$ which arose in applying (2.12).

The constant $K_{0}$ comes from estimates on simple random walk and is defined to be the sum of the various universal constants $c_{1}, c_{2}, \ldots$ occurring in the proof. We use $K_{1}$ to denote constants which are larger than $K_{0}$. In different occurrences $K_{1}$ may be different constants.

Proof of Theorem 4.1. (a) For fixed $\tau$ there are only finitely many $x \in \mathbb{Z}^{d}$ for which $x^{u} \partial_{z}^{v} \sum_{T=1}^{\tau} N_{\tau}(x, T) \varrho^{T}$ is nonzero. Hence the $L^{2}$ norm is the square root of the absolute value of a polynomial in $\varrho$, while the $L^{\infty}$ norm is the maximum of a finite family of functions, each of which is continuous in $\varrho$. Thus both norms are continuous in $\varrho$.

(b) It suffices to consider $\varrho \in\left(1, r_{\tau}\right]$ because $N_{\tau}(x, T) \leqq N_{0}(x, T)$ and for $\tau=0$ and $\varrho \leqq 1 P_{2}$ holds by Lemmas 3.2 and 3.4 , for some universal constant $K_{0}$. We first obtain the lower bound on $F_{\tau}(k, \varrho)$ mentioned above. Suppose that $P_{4}$ holds. By definition [Eq. (1.7)],

$$
\begin{aligned}
F_{\tau}(k, \varrho) & =1-\varrho D(k)-\Pi_{\tau}(k, \varrho) \\
& =1-\varrho-\Pi_{\tau}(0, \varrho)+\varrho(1-D(k))+\Pi_{\tau}(0, \varrho)-\Pi_{\tau}(k, \varrho) .
\end{aligned}
$$

By definition of $r_{\tau}$,

$$
F_{\tau}(0, \varrho)=1-\varrho-\Pi_{\tau}(0, \varrho) \geqq 0
$$

for $\varrho \in\left[0, r_{\tau}\right]$ with equality only for $\varrho=r_{\tau}$. 
Now by (2.13) and assumption,

$$
\left|\Pi_{\tau}(k, \varrho)-\Pi_{\tau}(0, \varrho)\right| \leqq K_{1} d^{-2} .
$$

The bound (4.3) is however not adequate for our present needs and we proceed to obtain a $k$ dependent bound. By (2.12) and assumption, the dominant behaviour of $\left|\partial_{k_{i} k_{j}}^{2} \Pi_{\tau}(k, \varrho)\right|$ is bounded by

$$
\begin{gathered}
c\left\|x_{i} x_{j} N_{\tau}^{(1)}\right\|_{2}\left\|N_{\tau}^{(1)}\right\|_{\infty}\left\|N_{\tau}^{(1)}\right\|_{2}+c\left\|x_{i} N_{\tau}^{(1)}\right\|_{2}\left\|x_{j} N_{\tau}^{(1)}\right\|_{2}\left\|N_{\tau}^{(1)}\right\|_{\infty} \\
\leqq K_{1}\left[d^{-s-1-\frac{1}{2}}+d^{-1-1-1}\right] \leqq K_{1}\left[\delta_{i j} d^{\frac{-5}{2}}+d^{-3}\right] .
\end{gathered}
$$

By (2.6), $\Pi_{\tau}(k, p)=\Pi_{\tau}(-k, p)$, and hence $\partial_{k_{i}} \Pi_{\tau}(0, \varrho)=0$. Therefore by Taylor's Theorem

$$
\begin{aligned}
\left|\Pi_{\tau}(k, \varrho)-\Pi_{\tau}(0, \varrho)\right| & =\left|\int_{0}^{1} d t(1-t) \frac{d^{2}}{d t^{2}} \Pi_{\tau}(t k, \varrho)\right| \\
& \leqq \frac{1}{2} \sup _{0 \leqq t \leqq 1}\left|\sum_{i, j=1}^{d} \partial_{k_{i} k_{j}}^{2} \Pi_{\tau}(t k, \varrho) k_{i} k_{j}\right| \\
& \leqq K_{1}\left[\sum_{i=1}^{d} d^{-5 / 2} k_{i}^{2}+\sum_{i \neq j} d^{-3}\left|k_{i} k_{j}\right|\right] \\
& \leqq K_{1}\left[d^{-5 / 2} k^{2}+d^{-3} \sum_{i, j=1}^{d}\left|k_{i} k_{j}\right| 1\right] \\
& \leqq K_{1}\left[d^{-5 / 2} k^{2}+d^{-3}(2 d)^{1 / 2} k^{2}\right]=K_{1} d^{-5 / 2} k^{2}
\end{aligned}
$$

using the Schwarz inequality in the last step. Using (3.8) and the above inequalities, and allowing $c$ to represent different constants in different occurrences, we have from (4.1) and (4.2)

$$
F_{\tau}(k, \varrho) \geqq \varrho(1-D(k))-K_{1} d^{-5 / 2} k^{2} \geqq\left(c d^{-1}-K_{1} d^{-5 / 2}\right) k^{2} \geqq c d^{-1} k^{2} \geqq c(1-D(k)) .
$$

Here $d$ may have to be taken larger; this remark will be left implicit in the sequel.

There are three conclusions to be checked:

Case 1: $\left\|\partial_{z}^{v} N_{\tau}^{(1)}(x, \varrho)\right\|_{\infty} \leqq 2 K_{0} d^{-1}, \quad v=0,1,2$,

Case 2: $\left\|\partial_{z}^{v} N_{\tau}^{(1)}(x, \varrho)\right\|_{2}^{2} \leqq 2 K_{0} d^{-1}, \quad v=0,1$,

Case 3: $\left\|x^{u} \partial_{z}^{v} N_{\tau}^{(1)}(x, \varrho)\right\|_{2} \leqq 2 K_{0} d^{-s}, \quad|u|=1,2, v=0,1$.

We consider these cases in turn.

Case 1. By differentiating $N_{\tau}^{(1)}(x, \varrho)=\sum_{T=1}^{\tau} N_{\tau}(x, T) \varrho^{T}$, it is seen that

$$
N_{\tau}^{(1)}(x, \varrho) \leqq \varrho \partial_{z} N_{\tau}^{(1)}(x, \varrho) \leqq \varrho N_{\tau}(x, T=1)+\varrho^{2} \partial_{z}^{2} N_{\tau}^{(1)}(x, \varrho) .
$$

Since $N_{\tau}(x, T=1) \leqq \frac{1}{2 d}$, it suffices to show that $\partial_{z}^{2} N_{\tau}^{(1)}(x, \varrho) \leqq($ universal const $) d^{-1}$. 
[As in the proof of Corollary 4.2(b), $\varrho=1+O\left(d^{-1}\right)$.] Now

$$
\begin{aligned}
0 & \leqq \partial_{z}^{2} N_{\tau}^{(1)}(x, \varrho) \leqq \partial_{z}^{2} N_{\tau}(x, \varrho)=(2 \pi)^{-d} \int d k e^{-i k x} \partial_{z}^{2} N_{\tau}(k, \varrho) \\
& =(2 \pi)^{-d} \int d k e^{-i k x}\left[\frac{\partial_{z}^{2} \Pi_{\tau}(k, \varrho)}{F_{\tau}(k, p)^{2}}+\frac{2\left(D(k)+\partial_{z} \Pi_{\tau}(k, \varrho)\right)^{2}}{F_{\tau}(k, p)^{3}}\right] \\
& =(2 \pi)^{-d} \int d k e^{-i k x}\left[\frac{\partial_{z}^{2} \Pi_{\tau}}{F_{\tau}^{2}}+\frac{2 D^{2}}{F_{\tau}^{3}}+\frac{4 D \partial_{z} \Pi_{\tau}}{F_{\tau}^{3}}+\frac{2\left(\partial_{z} \Pi_{\tau}\right)^{2}}{F_{\tau}^{3}}\right] .
\end{aligned}
$$

We examine each term on the right side, working from right to left. By assumption and (2.12), $\left|\partial_{z} \Pi_{\tau}\right| \leqq K_{1} d^{-1}$, and hence the fourth term is bounded by $K_{1} d^{-2}$, using (4.4) and (3.12). The third term is bounded using (4.4) and (3.12), the Schwarz inequality and Lemma $3.2(\mathrm{~b})$ as follows

$$
\left\|\frac{D \partial_{z} \Pi_{\tau}}{F_{\tau}^{3}}\right\|_{1} \leqq K_{1} d^{-1}\left\|\frac{D}{F_{\tau}^{2}}\right\|_{2}\left\|\frac{1}{F_{\tau}}\right\|_{2} \leqq K_{1} d^{-1} \cdot d^{-1 / 2} \cdot 1,
$$

where the middle factor is readied for application of Lemma 3.2(b) as follows:

$$
\left\|\frac{D}{F_{\tau}^{2}}\right\|_{2} \leqq C\left\|\frac{D}{(1-D)^{2}}\right\|_{2}=C\left\|\partial_{z} N_{0}(k, 1)\right\|_{2}=C\left\|\left.\partial_{z}\right|_{1} \sum_{T=1}^{\infty} N_{0}(x, T) z^{T}\right\|_{2} .
$$

The second term on the right-hand side of (4.5) is bounded by

$$
c\left\|\frac{2 D^{2}}{(1-D)^{3}}\right\|_{1}=c \partial_{z}^{2} N_{0}(x=0, z=1) \leqq C_{1} d^{-1}
$$

by Lemma $3.2($ b).

The first term on the right side of $(4.5)$ is more subtle. Write $\Pi_{\tau}(k, \varrho)=\Pi_{\tau}^{(1)}(k, \varrho)$ $+\Pi_{\tau}^{(>1)}(k, \varrho)$, where $\Pi_{\tau}^{(1)}(k, \varrho)$ is the contribution to the right side of $(2.6)$ from walks of the form $G_{1}$, i.e., from the lace consisting of a single bond. Evidently

$$
\partial_{z}^{2} \Pi_{\tau}^{(1)}(k, \varrho)=\partial_{z}^{2} \Pi_{\tau}^{(1)}(0, \varrho)<0,
$$

because $\prod_{s t \in L} U_{s t}=-1$ when $L=0 T$. Therefore

$$
(2 \pi)^{-d} \int d k e^{-i k x} \frac{\partial_{z}^{2} \Pi_{\tau}^{(1)}(k, \varrho)}{F_{\tau}(k, \varrho)^{2}}=\partial_{z}^{2} \Pi_{\tau}^{(1)}(0, \varrho)(2 \pi)^{-d} \int d k e^{-i k x} N_{\tau}(k, \varrho)^{2}<0,
$$

since the integral on the right side is the convolution in $x$-space of $N_{\tau}(x, \varrho)$ with itself and hence is positive. But the lace expansion can be used to bound $\left|\partial_{z}^{2} \Pi_{\tau}^{(>1)}(k, \varrho)\right|$ by $K_{1} d^{-2}$, since the first term on the right side of $(2.12)$ will be absent. Putting it all together gives

$$
0 \leqq \partial_{z}^{2} N_{\tau}(x, \varrho) \leqq C_{1} d^{-1}+O\left(d^{-3 / 2}\right) .
$$

Case 2. As noted in Case 1, $N_{\tau}^{(1)}(x, \varrho) \leqq \varrho \partial_{z} N_{\tau}^{(1)}(x, \varrho)$, and it suffices to consider $v=1$. Now

$$
\begin{aligned}
\left\|\partial_{z} N_{\tau}^{(1)}(x, \varrho)\right\|_{2}^{2} & \leqq\left\|\partial_{z} N_{\tau}(x, \varrho)\right\|_{2}^{2}=\left\|\partial_{z} N_{\tau}(k, \varrho)\right\|_{2}^{2} \\
& =(2 \pi)^{-d} \int d k \frac{\left(D(k)+\partial_{z} \Pi_{\tau}(k, \varrho)\right)^{2}}{F_{\tau}(k, \varrho)^{4}} \\
& =(2 \pi)^{-d} \int d k \frac{D^{2}+2 D \partial_{z} \Pi_{\tau}+\left(\partial_{z} \Pi_{\tau}\right)^{2}}{F_{\tau}^{4}} .
\end{aligned}
$$


By (4.4) and Lemma 3.2(b) the first term is bounded by

$$
C\left\|\frac{D}{(1-D)^{2}}\right\|_{2}^{2}=C\left\|\partial_{z} N_{0}(k, 1)\right\|_{2}^{2} \leqq C_{2} d^{-1}
$$

By assumption and (2.12) $\left|\partial_{z} \Pi_{\tau}(k, \varrho)\right| \leqq K_{1} d^{-1}$, so the third term is $O\left(d^{-2}\right)$. The second term is less than

$$
\frac{K_{1}}{d}\left\|\frac{D}{(1-D)^{4}}\right\|_{1} \leqq \frac{K_{1}}{d}\left\|\frac{D}{(1-D)^{2}}\right\|_{2}\left\|\frac{1}{(1-D)^{2}}\right\|_{2} \leqq K_{1} d^{-3 / 2}
$$

by Lemma 3.2 (b) and (3.12). Therefore

$$
\left\|\partial_{z} N_{\tau}^{(1)}(x, \varrho)\right\|_{2}^{2} \leqq C_{2} d^{-1}+O\left(d^{-3 / 2}\right) .
$$

Case 3. Since $\left|x_{i}\right| \leqq x_{i}^{2}$, it suffices to take $|u|=2$, and as in the previous case it suffices to take $v=1$. Now

$$
\left\|x_{i} x_{j} \partial_{z} N_{\tau}^{(1)}(x, \varrho)\right\|_{2} \leqq\left\|x_{i} x_{j} \partial_{z} N_{\tau}(x, \varrho)\right\|_{2}=\left\|\partial_{k_{i} k_{j}}^{2} \partial_{z} N_{\tau}(k, \varrho)\right\|_{2} .
$$

Using the obvious abbreviations,

$$
\partial_{k_{2} k_{j}}^{2} \partial_{z} N_{\tau}(k, p)=-\frac{F_{i j z}}{F^{2}}+\frac{2 F_{i z} F_{j}}{F^{3}}+\frac{2 F_{j z} F_{i}}{F^{3}}+\frac{2 F_{z} F_{i j}}{F^{3}}-\frac{6 F_{z} F_{i} F_{j}}{F^{4}}
$$

with

$$
\begin{array}{rlrl}
F_{i} & =\frac{\varrho}{d} \sin k_{i}-\Pi_{i}, & F_{z} & =-D-\Pi_{z}, \\
F_{i j} & =\frac{\varrho}{d} \delta_{i j} \cos k_{i}-\Pi_{i j}, \quad F_{z i}=\frac{1}{d} \sin k_{i}-\Pi_{z i}, \\
F_{i j z} & =\frac{1}{d} \delta_{i j} \cos k_{i}-\Pi_{i j z} .
\end{array}
$$

We number the terms on the right side of (4.6) as $1-5$ and look at their $L^{2}$ norms, working from left to right. We show that these terms behave like the corresponding terms on the right side of (3.13). To begin,

$$
\|1\|_{2} \leqq C\left\|\frac{D_{i j}}{(1-D)^{2}}\right\|_{2}+C\left\|\Pi_{i j z}\right\|_{\infty}\left\|\frac{1}{(1-D)^{2}}\right\|_{2} \leqq C_{3} \delta_{i j} d^{-1}+K_{1} d^{-5 / 2}
$$

where $\left|\Pi_{i j z}\right|$ is bounded as $\left|\Pi_{i j}\right|$ was bounded under (4.3). The terms 2 and 3 are bounded the same way:

$$
\begin{aligned}
\|2\|_{2} \leqq & \left\|\frac{\left(D_{i}+\Pi_{z i}\right)\left(\varrho D_{j}+\Pi_{j}\right)}{(1-D)^{3}}\right\|_{2} \\
\leqq & \left\|\frac{D_{i} D_{j}}{(1-D)^{3}}\right\|_{2}+C\left\|\Pi_{z i}\right\|_{\infty}\left\|\frac{D_{j}}{(1-D)^{3}}\right\|_{2}+C\left\|\Pi_{j}\right\|_{\infty}\left\|\frac{D_{i}}{(1-D)^{3}}\right\|_{2} \\
& +C\left\|\Pi_{z i}\right\|_{\infty}\left\|\Pi_{j}\right\|_{\infty}\left\|\frac{1}{(1-D)^{3}}\right\|_{2} \\
\leqq & C_{4} d^{-2}+O\left(d^{-7 / 2}\right)
\end{aligned}
$$


since $\left|D_{i}\right| \leqq d^{-1},\left\|(1-D)^{-3}\right\|_{2} \leqq C$ by (3.12), and $\left|\Pi_{z i}\right|$ and $\left|\Pi_{j}\right|$ are $O\left(d^{-5 / 2}\right)$ by the same argument used to bound $\left|\Pi_{i j z}\right|$ above. Similarly,

$$
\begin{aligned}
\|4\|_{2} & \leqq C\left\|\frac{\left(D+O\left(d^{-1}\right)\right)\left(\varrho D_{i j}+O\left(d^{-5 / 2}\right)\right)}{(1-D)^{3}}\right\|_{2} \\
& \leqq C_{5} \delta_{i j} d^{-1}+O\left(d^{-5 / 2}\right)+\delta_{i j} O\left(d^{-2}\right)+O\left(d^{-7 / 2}\right) .
\end{aligned}
$$

Finally,

$$
\|5\|_{2} \leqq C\left\|\frac{\left(D+O\left(d^{-1}\right)\right)\left(\varrho D_{i}+O\left(d^{-5 / 2}\right)\right)\left(\varrho D_{j}+O\left(d^{-5 / 2}\right)\right)}{(1-D)^{4}}\right\|_{2} \leqq C_{6} d^{-2}+O\left(d^{-3}\right) .
$$

This completes the proof of Theorem 4.1.

We now increase the domain of analyticity of $\Pi_{\tau}(k, z)$ to $z \in D_{\tau}\left(\frac{1}{2}\right)=\{z:|z|$ $\left.\leqq r_{\tau}\left(1+\frac{1}{2} \tau^{-1} \ln \tau\right)\right\}$ at the expense of one $z$-derivative in the estimates.

Theorem 4.3. There is a positive integer $d_{0}$ such that for $d \geqq d_{0} \Pi_{\tau}(k, z)$ and $\partial_{k}^{u} \Pi_{\tau}(k, z),|u| \leqq 2$, are analytic in $z \in D_{\tau}\left(\frac{1}{2}\right)$ with $\left|\partial_{z}^{v} \Pi_{\tau}(k, z)\right| \leqq$ const $^{-1}, v=0,1$, and $\left|\partial_{k}^{u} \Pi_{\tau}(k, z)\right| \leqq$ const $d^{-5 / 2},|u|=1,2$, with the constants independent of $\tau$ and $z \in D_{\tau}\left(\frac{1}{2}\right)$.

Proof. For $|z| \leqq r_{\tau}\left(1+\frac{1}{2} \tau^{-1} \ln \tau\right)$,

$$
\begin{aligned}
\left|N_{\tau}^{(1)}(x, z)\right| & =\left|\sum_{T=1}^{\tau} N_{\tau}(x, T) z^{T}\right| \leqq \sum_{T=1}^{\tau} N_{\tau}(x, T)\left[r_{\tau}\left(1+\frac{1}{2} \tau^{-1} \ln \tau\right)\right]^{T} \\
& =r_{\tau} \sum_{T=1}^{\tau} N_{\tau}(x, T) T r_{\tau}^{T-1} T^{-1}\left(1+\frac{1}{2} \tau^{-1} \ln \tau\right)^{T}
\end{aligned}
$$

It is easy to check that $T^{-1}\left(1+\frac{1}{2} \tau^{-1} \ln \tau\right)^{T}$ is bounded uniformly in $\tau$ and $T$ for $1 \leqq T \leqq \tau$. Since $r_{\tau}=1-\Pi_{\tau}\left(0, r_{\tau}\right)$ is also uniformly bounded we have

$$
\left|N_{\tau}^{(1)}(x, z)\right| \leqq c \partial_{z} N_{\tau}^{(1)}\left(x, r_{\tau}\right)
$$

Similarly

and

$$
\left|\partial_{z} N_{\tau}^{(1)}(x, z)\right| \leqq c \partial_{z}^{2} N_{\tau}^{(1)}\left(x, r_{\tau}\right)+N_{\tau}(x, T=1)
$$

$$
\left|\partial_{z}^{v} N_{\tau}^{(0)}(x, z)\right| \leqq \delta_{x, 0} \delta_{v, 0}+c \partial_{z}^{v+1} N_{\tau}^{(1)}\left(x, r_{\tau}\right)+\delta_{v, 1} N_{\tau}(x, T=1), \quad v=0,1
$$

Now we use (2.12) and (4.7-9) to estimate the derivatives of $\Pi_{\tau}$ occurring in the statement of the theorem. For example,

$$
\begin{aligned}
\left|\partial_{z} \Pi_{\tau}(x, z)\right| \leqq & c\left\|\partial_{z} N_{\tau}^{(1)}(x,|z|)\right\|_{\infty} \\
& \times\left[1+\sum_{N=2}^{\infty}(2 N-1)\left\|N_{\tau}^{(1)}(x,|z|)\right\|_{2}^{N}\left\|N_{\tau}^{(0)}(x,|z|)\right\|_{2}^{N-2}\right] \\
\leqq & c\left[\left\|\partial_{z}^{2} N_{\tau}^{(1)}\left(x, r_{\tau}\right)\right\|_{\infty}+\frac{1}{2 d}\right]\left[1+\sum_{N=2}^{\infty}(2 N-1) c^{2 N-2}\left\|\partial_{z} N_{\tau}^{(1)}\left(x, r_{\tau}\right)\right\|_{2}^{N}\right. \\
& \left.\times\left(1+\left\|\partial_{z} N_{\tau}^{(1)}\left(x, r_{\tau}\right)\right\|_{2}\right)^{N-2}\right] \leqq O\left(d^{-1}\right)
\end{aligned}
$$

by Corollary 4.2. The estimates for $\left|\Pi_{\tau}(k, z)\right|$ and $\left|\partial_{k}^{u} \Pi_{\tau}(k, z)\right|$ are similar. 


\section{Proof of Theorem 1.1}

In this section we prove Theorem 1.1 using the results of the preceding sections. The approach is similar to that used in [3]. The first observation is the following lemma, in the statement of which $c$ is a universal constant.

Lemma 5.1. For $k^{2} \leqq c d \tau^{-1} \ln \tau$ and $d \geqq d_{0}, N_{\tau}(k, z)$ has a simple pole at $r_{\tau}(k) \in D_{\tau}(1 / 4)$ and is otherwise analytic in $D_{\tau}(1 / 2)$. The pole $r_{\tau}(k)$ is twice differentiable in $k$.

Proof. Singularities of $N_{\tau}$ correspond to zeroes of $F_{\tau}$. Zeroes of $F_{\tau}$ occur in complex conjugate pairs since $\overline{F_{\tau}(k, z)}=F_{\tau}(k, \bar{z})$ [the right side of $(2.6)$ is unchanged by the replacement of $k$ by $-k]$. Suppose $z_{1}$ and $z_{2}$ are two zeroes of $F_{\tau}(k, z)$. Then

$$
\left(z_{2}-z_{1}\right) D(k)=\Pi_{\tau}\left(k, z_{1}\right)-\Pi_{\tau}\left(k, z_{2}\right)=-\left(z_{2}-z_{1}\right) \int_{0}^{1} \partial_{z} \Pi_{\tau}\left(k, t z_{2}+(1-t) z_{1}\right) d t .
$$

For small $k^{2}, D(k) \simeq 1$ while for large $d,\left|\partial_{z} \Pi_{\tau}\right| \leqq K d^{-1}$, so (5.1) is only possible if $z_{1}=z_{2}$. Thus for small $k^{2}, F_{\tau}(k, z)$ has at most one zero in $D_{\tau}(1 / 2)$, which must be real.

For $k=0, r_{\tau}$ is a simple zero of $F_{\tau}(0, z)$. In fact

$$
\begin{aligned}
F_{\tau}(0, z) & =F_{\tau}(0, z)-F_{\tau}\left(0, r_{\tau}\right)=-\left[\left(z-r_{\tau}\right)+\Pi_{\tau}(0, z)-\Pi_{\tau}\left(0, r_{\tau}\right)\right] \\
& =-\left(z-r_{\tau}\right)\left[1+\int_{0}^{1} \partial_{z} \Pi_{\tau}\left(0, t z+(1-t) r_{\tau}\right) d t\right]
\end{aligned}
$$

and the second factor is nonzero in $D_{\tau}(1 / 2)$ by Theorem 4.3. By the implicit function theorem, for small $k^{2}, F_{\tau}(k, z)$ has a simple zero $r_{\tau}(k)$ with $r_{\tau}(k) \in D_{\tau}(1 / 2)$. Derivatives of $r_{\tau}(k)$ are obtained by differentiating the equation $F_{\tau}\left(k, r_{\tau}(k)\right)=0$. The first derivative is

$$
\partial_{i} r_{\tau}(k)=\frac{r_{\tau}(k) d^{-1} \sin k_{i}-\partial_{i} \Pi_{\tau}\left(k, r_{\tau}(k)\right)}{D(k)+\partial_{z} \Pi_{\tau}\left(k, r_{\tau}(k)\right)} \approx \frac{r_{\tau}(k)}{d} k_{i}
$$

for small $k^{2}$, using Theorem 4.3, Taylor's theorem, and the fact that $\Pi_{\tau}(k, z)$ is even in $k_{i}$ to see that $\partial_{i} \Pi_{\tau}\left(k, r_{\tau}(k)\right)=O\left(d^{-5 / 2}\right) k_{i}$. The formula (5.2) is valid as long as $r_{\tau}(k) \in D_{\tau}(1 / 2)$. Now $r_{\tau}(t k)$ is increasing in $t>0$ since

Therefore

$$
\frac{d}{d t} r_{\tau}(t k)=\sum_{i=1}^{d} \partial_{i} r_{\tau}(t k) k_{i} \approx r_{\tau}(t k) d^{-1} k^{2}>0
$$

$$
r_{\tau}(k)=r_{\tau}+\int_{0}^{1} \frac{d}{d t} r_{\tau}(t k) d t \leqq r_{\tau}+c^{\prime} r_{\tau}(k) d^{-1} k^{2}
$$

and so

$$
r_{\tau}(k) \leqq r_{\tau}\left(1-c^{\prime} d^{-1} k^{2}\right)^{-1} \leqq r_{\tau}\left(1+c^{\prime \prime} d^{-1} k^{2}\right) \leqq r_{\tau}\left(1+\frac{1}{4} \tau^{-1} \ln \tau\right),
$$

provided $k^{2} \leqq c d \tau^{-1} \ln \tau$.

The second derivatives of $r_{\tau}(k)$ are obtained from

$$
F_{i j}+F_{i z} r_{j}+F_{j z} r_{i}+F_{z} r_{i j}+F_{z z} r_{i} r_{j}=0 .
$$


Theorem 1.1. There is a constant $d_{0} \geqq 5$ such that for $d \geqq d_{0},\left\langle\omega(T)^{2}\right\rangle=D T$ $+O\left(T^{1 / 2} \ln T\right)$ as $T \rightarrow \infty$, with $D>1$.

Proof. Let $C$ be the circle of radius $1 / 2$ centred at the origin, oriented counterclockwise. Then for $k^{2} \leqq c d \tau^{-1} \ln \tau$ it follows from Lemma 5.1 and the Residue Theorem that

$$
\begin{aligned}
N_{\tau}(k, T) & =\frac{1}{2 \pi i} \int_{C} N_{\tau}(k, z) \frac{d z}{z^{T+1}} \\
& =-\operatorname{Res}_{z=r_{\tau}(k)} N_{\tau}(k, z) z^{-(T+1)}+\frac{1}{2 \pi i} \int_{\partial D_{\tau}(1 / 2)} N_{\tau}(k, z) \frac{d z}{z^{T+1}} \\
& =r_{\tau}(k)^{-(T+1)}\left[-\partial_{z} F_{\tau}\left(k, r_{\tau}(k)\right)^{-1}+\frac{1}{2 \pi i} \int_{\partial D_{\tau}(1 / 2)} N_{\tau}(k, z)\left(\frac{r_{\tau}(k)}{z}\right)^{T+1} d z\right] .
\end{aligned}
$$

Since $\partial_{k_{i}} F_{\tau}$ and $\partial_{k_{i}} r_{\tau}$ both vanish at $k=0$, applying $\nabla_{k}^{2}$ to (5.4) and evaluating at $k=0$ gives

$$
\begin{aligned}
\nabla_{k}^{2} N_{\tau}(0, T)= & -\left.\nabla_{k}^{2}\right|_{0}\left[r_{\tau}(k)^{-T-1}\right] \partial_{z} F_{\tau}\left(0, r_{\tau}\right)^{-1} \\
& -\left.r_{\tau}^{-(T+1)} \nabla_{k}^{2}\right|_{0}\left[\partial_{z} F_{\tau}\left(k, r_{\tau}(k)^{-1}\right]\right. \\
& +\frac{1}{2 \pi i} \int_{\partial D_{\tau}(1 / 2)} \nabla_{k}^{2} N_{\tau}(0, z) \frac{d z}{z^{T+1}}
\end{aligned}
$$

Now

and

$$
\left.\nabla_{k}^{2}\right|_{0} r_{\tau}(k)^{-T-1}=-(T+1) r_{\tau}^{-T-2} \nabla_{k}^{2} r_{\tau}(0)
$$

$$
\left.\nabla_{k}^{2}\right|_{0}\left[\partial_{z} F_{\tau}\left(k, r_{\tau}(k)\right)\right]^{-1}=-\left[\partial_{z} F_{\tau}\left(0, r_{\tau}\right)\right]^{-2}\left[\nabla_{k}^{2} \partial_{z} F_{\tau}\left(0, r_{\tau}\right)+\partial_{z}^{2} F_{\tau}\left(0, r_{\tau}\right) \nabla_{k}^{2} r_{\tau}(0)\right] .
$$

By Corollary 4.2, $\partial_{z} F_{\tau}\left(0, r_{\tau}\right)=-1+O\left(d^{-1}\right)$, and $\partial_{z}^{2} F_{\tau}\left(0, r_{\tau}\right)$ and $\nabla_{k}^{2} \partial_{z} F_{\tau}\left(0, r_{\tau}\right)$ are bounded uniformly in $\tau$. Setting $k=0$ in (5.3) gives

$$
\nabla_{k}^{2} r_{\tau}(0)=-\left[\partial_{z} F_{\tau}\left(0, r_{\tau}\right)\right]^{-1} \nabla_{k}^{2} F_{\tau}\left(0, r_{\tau}\right),
$$

which is similarly bounded uniformly in $\tau$.

Putting $\tau=T$ and using (1.5), (5.4-5) and the preceding paragraph leads to

$$
\begin{aligned}
\left\langle\omega(T)^{2}\right\rangle_{T}= & {\left[(T+1) D_{T}+O(1)+O(1) \int_{\partial D_{T}\left(\frac{1}{2}\right)} \nabla_{k}^{2} N_{T}(0, z)\left(\frac{r_{T}}{z}\right)^{T+1} d z\right] } \\
& \times\left[1+O(1) \int_{\partial D_{\tau\left(\frac{1}{2}\right)}} N_{\tau}(0, z)\left(\frac{r_{T}}{z}\right)^{T+1} d z\right]^{-1},
\end{aligned}
$$

where $D_{\tau}=r_{\tau}^{-1} \nabla_{k}^{2} r_{\tau}(0)$.

To estimate the integrals occurring on the right side of (5.9) we first observe that for $z \in \partial D_{T}(1 / 2)$,

$$
\left|\frac{r_{T}}{z}\right|^{T+1}=\left(1+\frac{1}{2} T^{-1} \ln T\right)^{-(T+1)} \leqq c T^{-\frac{1}{2}}
$$


Therefore

$$
\left|\int_{\partial D_{T}(1 / 2)} \partial_{k_{i}}^{u} N_{T}(0, z)\left(\frac{r_{T}}{z}\right)^{T+1} d z\right| \leqq c T^{-\frac{1}{2}} \int_{\partial D_{T}(1 / 2)}\left|\partial_{k_{i}}^{u} N_{T}(0, z)\right||d z| .
$$

It follows from the equation above (5.2) that for some constant $c$

$$
\left|N_{T}(0, z)\right| \leqq c\left|z-r_{T}\right|^{-1}
$$

and hence for $u=0$ the right side of (5.10) is bounded by $c T^{-\frac{1}{2}} \ln T$. For $u=2$ we have

$$
\partial_{k_{i}}^{2} N_{T}(0, z)=\frac{-\partial_{k_{r}}^{2} F_{T}(0, z)}{F_{T}(0, z)^{2}} .
$$

By (5.11) and Theorem 4.3 the right side of (5.12) is bounded by $c d^{-1}\left|z-r_{T}\right|^{-2}$, and so the right side of $(5.10)$ is bounded by $C T^{-\frac{1}{2}} d^{-1}\left(T^{-1} \ln T\right)^{-1} \leqq c d^{-1} T^{\frac{1}{2}}$, when $u=2$.

Substituting these estimates into (5.9) gives

$$
\left\langle\omega(T)^{2}\right\rangle_{T} \leqq D_{T} T\left(1+O\left(T^{-\frac{1}{2}} \ln T\right)\right) .
$$

To complete the proof we show that $D_{T}=D+O\left(T^{-1}\right)$, with $D>1$. Consider two different memories $\sigma<\tau$. From the fact that $F_{\sigma}\left(k, r_{\sigma}(k)\right)=0$ and $F_{\tau}\left(k, r_{\tau}(k)\right)=0$, it follows that

$$
\left(r_{\tau}(k)-r_{\sigma}(k)\right) D(k)=-\left[\Pi_{\tau}\left(k, r_{\tau}(k)\right)-\Pi_{\sigma}\left(k, r_{\sigma}(k)\right)\right] .
$$

Setting $k=0$ gives

$$
r_{\tau}-r_{\sigma}=-\left[\Pi_{\tau}\left(0, r_{\tau}\right)-\Pi_{\tau}\left(0, r_{\sigma}\right)\right]-\left[\Pi_{\tau}\left(0, r_{\sigma}\right)-\Pi_{\sigma}\left(0, r_{\sigma}\right)\right] .
$$

This implies that

$$
\left(1+\partial_{z} \Pi_{\tau}\left(0, r^{*}\right)\right)\left(r_{\tau}-r_{\sigma}\right)=-\delta \Pi\left(0, r_{\sigma}\right),
$$

where $r^{*} \in\left(r_{\sigma}, r_{\tau}\right)$ and $\delta \Pi=\Pi_{\tau}-\Pi_{\sigma}$. By Theorem 4.3 the coefficient of $r_{\tau}-r_{\sigma}$ is bounded below by a positive constant. The absolute value of the right side is bounded using (2.14), where in (2.14) the $L^{\infty}$ norm is coordinated with the $\delta N$. Each term on the right side of (2.14) has a factor

$$
\begin{aligned}
\left\|\delta N\left(x, r_{\sigma}\right)\right\|_{\infty} & \leqq\left\|\sum_{T=\sigma / 6}^{\infty} N_{\sigma}(x, T) r_{\sigma}^{T}\right\|_{\infty} \leqq 6 \sigma^{-1}\left\|\sum_{T=\sigma / 6}^{\infty} N_{\sigma}(x, T) T r_{\sigma}^{T-1}\right\|_{\infty} r_{\sigma} \\
& \leqq 6 r_{\sigma} \sigma^{-1}\left\|\partial_{z} N_{\sigma}\left(x, r_{\sigma}\right)\right\|_{\infty} \leqq \operatorname{const} \sigma^{-1},
\end{aligned}
$$

since $\left\|\partial_{z} N_{\sigma}\left(x, r_{\sigma}\right)\right\|_{\infty}$ is bounded by the argument used in Case 1 of the proof of Theorem 4.1 (b). Therefore

$$
r_{\tau}-r_{\sigma} \leqq c \sigma^{-1}
$$

uniformly in $\tau$ and so

$$
r_{\infty}-r_{T} \leqq c T^{-1}
$$


The Laplacian is treated similarly. Differentiation of Eq. (5.14) gives

$$
\begin{aligned}
\nabla_{k}^{2} r_{\tau}(0) & -\nabla_{k}^{2} r_{\sigma}(0)=r_{\tau}-r_{\sigma}-\left[\nabla_{k}^{2} \Pi_{\tau}\left(0, r_{\tau}\right)-\nabla_{k}^{2} \Pi_{\sigma}\left(0, r_{\sigma}\right)\right] \\
- & {\left[\partial_{z} \Pi_{\tau}\left(0, r_{\tau}\right) \nabla_{k}^{2} r_{\tau}(0)-\partial_{z} \Pi_{\sigma}\left(0, r_{\sigma}\right) \nabla_{k}^{2} r_{\sigma}(0)\right] . }
\end{aligned}
$$

Using Taylor's Theorem as above,

$$
\nabla_{k}^{2} \Pi_{\tau}\left(0, r_{\tau}\right)-\nabla_{k}^{2} \Pi_{\sigma}\left(0, r_{\sigma}\right)=\partial_{z} \nabla_{k}^{2} \Pi_{\tau}\left(0, r^{*}\right)\left(r_{\tau}-r_{\sigma}\right)+\nabla_{k}^{2} \delta \Pi\left(0, r_{\sigma}\right),
$$

where $r^{*} \in\left(r_{\sigma}, r_{\tau}\right)$. The first factor of the first term on the right side is uniformly bounded by Corollary 4.2 (b), and hence the first term is bounded by $c \sigma^{-1}$, by (5.15). The second term on the right side is bounded in the same manner that $\delta \Pi\left(0, r_{\sigma}\right)$ was bounded, apart from the fact that an $L^{2}$ norm is associated with any subwalk involving an $x^{u_{\imath}}$ with $u_{i} \neq 0$, because in Corollary 4.2 (a) it was the $L^{2}$ norm of $k$ derivatives that was bounded. The final term on the right side of (5.17) can be analyzed in a similar fashion with the result that

$$
\left|\nabla_{k}^{2} r_{\tau}(0)-\nabla_{k}^{2} r_{\sigma}(0)\right| \leqq c \sigma^{-1}
$$

uniformly in $\tau$. Putting $\sigma=T$ and letting $\tau \rightarrow \infty$ gives

$$
\left|\nabla_{k}^{2} r_{\infty}(0)-\nabla_{k}^{2} r_{T}(0)\right| \leqq c T^{-1}
$$

which together with (5.16) implies

$$
D_{T}=\frac{\nabla_{k}^{2} r_{T}(0)}{r_{T}}=\frac{\nabla_{k}^{2} r_{\infty}(0)+O\left(T^{-1}\right)}{r_{\infty}+O\left(T^{-1}\right)}=D+O\left(T^{-1}\right),
$$

where $D=r_{\infty}^{-1} \nabla_{k}^{2} r_{\infty}(0)$.

We now complete the proof of Theorem 1.1 by showing that $D>1$. It suffices to show that $D_{\tau}>\beta$ where $\beta>1$, for all $\tau$. By (5.8),

$$
D_{\tau}=-r_{\tau}^{-1} \frac{\nabla_{k}^{2} F_{\tau}\left(0, r_{\tau}\right)}{\partial_{z} F_{\tau}\left(0, r_{\tau}\right)}=\frac{1-r_{\tau}^{-1} \nabla_{k}^{2} \Pi_{\tau}\left(0, r_{\tau}\right)}{1+\partial_{z} \Pi_{\tau}\left(0, r_{\tau}\right)}
$$

For large $d$ the dominant contribution to $\partial_{z} \Pi_{\tau}\left(0, r_{\tau}\right)$ comes from the $G_{1}$ diagrams in the lace expansion. These diagrams evidently give a negative contribution which is bounded away from zero uniformly in $\tau$. In fact the contribution from these diagrams is less than $-r_{\tau} d^{-1}$, which is the $T=2$ term in the derivative with respect to $z$ of (2.6), corresponding to walks which take one step away from the origin and then immediately return to the origin. Using the lace expansion to bound the difference between $\partial_{z} \Pi_{\tau}\left(0, r_{\tau}\right)$ and the $G_{1}$ diagrams gives the bound (2.12) without the first term. By Corollary 4.2 this difference is $O\left(d^{-2}\right)$, and hence for large $d$ and some $a \in(0,1)$,

$$
1+\partial_{z} \Pi_{\tau}\left(0, r_{\tau}\right)=1-\left|O\left(d^{-1}\right)\right| \leqq a<1
$$

Similarly the dominant contribution to $\nabla_{k}^{2} \Pi_{\tau}\left(0, r_{\tau}\right)$ comes from the lace $>$ corresponding to the diagrams $G_{2}$. This contribution is evidently negative and less than $d$ times the $T=3$ term with the lace three step walks of the form $\ominus$, i.e., less than $-2 d^{2}\left(\frac{r_{\tau}}{2 d}\right)^{3}<-(1 / 4) d^{-1}$. We now 
argue that the contribution due to all other laces is $O\left(d^{-5 / 2}\right)$. In fact apart from , which corresponds to walks with $\omega(T)=0$ and hence does not contribute to $k$-derivatives, all other laces correspond to walks which can be broken up into subwalks with at least five of the subwalks consisting of at least one step. Estimating the difference between $\nabla_{k}^{2} \Pi_{\tau}\left(0, r_{\tau}\right)$ and its dominant contribution as in (2.12) gives the bound $O\left(d^{-5 / 2}\right)$, since by Corollary 4.2 two subwalks can be bounded by $O\left(d^{-1}\right)$ and the remainder are $O\left(d^{-1 / 2}\right)$, while there are $d$ terms in the Laplacian. Therefore for some $b>0, \nabla_{k}^{2} \Pi_{\tau}\left(0, r_{\tau}\right) \leqq-\left|O\left(d^{-1}\right)\right| \leqq-b<0$ uniformly in $\tau$.

From this and (5.18-19) it follows that

$$
D_{\tau} \geqq \frac{1+b}{a}>1
$$

since $a<1$ and $b>0$. This completes the proof of Theorem 1.1.

Acknowledgement. It is a pleasure to thank David Brydges for introducing me to this problem and for providing insights into its solution in many conversations.

\section{References}

1. Brydges, D.: A short course on cluster expansions. Lectures given at the 1984 Les Houches summer school (to be published)

2. Brydges, D., Fröhlich, J., Sokal, A.: A new proof of the existence and nontriviality of the continuum $\phi_{2}^{4}$ and $\phi_{3}^{4}$ quantum field theories. Commun. Math. Phys. 91, 141-186 (1983)

3. Brydges, D., Spencer, T.: Self-avoiding walk in 5 or more dimensions. Commun. Math. Phys. 97, 125-148 (1985)

4. Domb, C.: Self-avoiding walk on lattices. In: Stochastic processes in chemical physics. Shuler, K.E. (ed.), pp. 229-260. New York: Wiley 1969

5. Kesten, H.: On the number of self-avoiding walks. II. J. Math. Phys. 5, 1128-1137 (1964)

6. Lawler, G.: Gaussian behaviour of loop-erased self-avoiding random walk in four dimensions. Duke Math. J. 53, 249-269 (1986), and A self-avoiding random walk. Duke Math. J. 47, 655-693 (1980)

7. Bovier, A., Felder, G., Fröhlich, J.: On the critical properties of the Edwards and the selfavoiding walk model of polymer chains. Nucl. Phys. B 230 [FS10], 119-147 (1984)

8. Aragão de Carvalho, C., Caracciolo, S., Fröhlich, J.: Polymers and $g|\phi|^{4}$ theory in four dimensions. Nucl. Phys. B 215 [FS7], 209-248 (1983)

Communicated by J. Fröhlich

Received October 1, 1986; in revised form January 12, 1987 